Рrоoм, H. (1955). J. gen. Microbiol. 13, 170-175.

\title{
Amine Production and Nutrition in the Providence Group
}

\author{
BY H. PROOM \\ Wellcome Research Laboratories (Biological Division), Beckenham, Kent
}

\begin{abstract}
SUMMARY : Fifty-six strains of non-lactose fermenting coliform organisms belonging to the Providence group were obtained from the National Collection of Type Cultures and examined for their nutritional requirements. Fifty-five of these strains formed a nutritionally homogeneous group. The simplest medium supporting growth was a basal salt medium with added glucose, glutamic acid and cystine. Pantothenate usually improved growth and appeared to be nearly essential for two strains. The remaining strain possessed the nutritional pattern of Proteus morganii and in this and other characters appeared to be a typical strain of that species. Twenty-two of the fifty-five strains gave a positive amine test using the ninhydrin test-paper technique; previously only Proteus species in the family Enterobacteriaceae had been observed to produce amines. The classification of the Providence group is discussed in the light of these findings.
\end{abstract}

Proom \& Woiwod (1951) showed that the ability to produce amines by decarboxylation of certain amino acids was a constant and characteristic property of Proteus species. All of 47 strains of Proteus vulgaris, 75 strains of $\boldsymbol{P}$. mirabilis and 10 strains of $\boldsymbol{P}$. morganii were found to produce amines, whereas none of 257 strains representing other species of Enterobacteriaceae possessed this property. Although a wide selection of Enterobacteriaceae, including many strains of uncertain taxonomic status, were examined, the selection did not include any of the anaerogenic paracolon organisms classified by Kauffmann (1951) in the Providence group.

When Proteus morganii, strain 1721, of Stamp \& Stone (1944) was examined, it was found to produce amines but did not decompose urea or require nicotinic acid as an essential metabolite. It was not therefore a typical Proteus species, and Kauffmann (1951) placed this strain in the Providence group. In consequence of this anomalous finding some Providence strains were obtained from the National Collection of Type Cultures and examined in particular for their ability to produce amines and for their nutritional requirements. The results are reported here and discussed in relation to classification.

\section{MATERIALS AND METHODS}

Strains. Fifty-six Providence strains were obtained from the National Collection of Type Cultures (NCTC 8993-9000 and NCTC 9190-9237). These strains were received by the National Collection from Dr W. H. Ewing.

Fermentation tests. Cultures were examined for their ability to ferment glucose, lactose, maltose, mannitol and sucrose. Fermentation tests were carried out in $\frac{1}{4} \mathrm{oz}$. screw-capped bottles containing $c .3 \mathrm{ml}$. of $1.0 \%$ peptone 
water $+\mathbf{0 . 5} \%$ of the relevant carbohydrate, with bromcresol purple as indicator. The cultures were examined at intervals up to 14 days.

Indole reaction was determined by Kovács's (1928) method using amyl alcohol as the solvent.

Voges-Proskauer reaction. Cultures were grown in glucose peptone water and the reaction determined by O'Meara's (1931) technique.

Methyl red reaction was determined in $\frac{1}{4}$ oz. screw-capped bottles containing c. $3 \mathrm{ml}$. glucose phosphate broth and the indicator added after 3 days incubation at $37^{\circ} \mathrm{C}$.

Liquefaction of gelatin was determined in nutrient gelatin by incubation at $37^{\circ}$ for 7 days, the tubes being cooled before reading the tests.

Decomposition of urea was determined in Christensen's (1946) medium and the results read after 4 and $24 \mathrm{hr}$.

$\mathrm{H}_{2} \mathrm{~S}$ production was determined by growth in $10 \mathrm{ml}$. nutrient broth contained in cotton-wool plugged test tubes with a strip of lead acetate paper as indicator; the results were read at intervals up to 6 days.

Phenylpyruvic acid reaction was determined by the method described by Henricksen (1950).

Amine production was determined by the ninhydrin test-paper technique described by Proom \& Woiwod (1951).

Minimal nutritional requirements. The methods used were similar to those described by Knight \& Proom (1950). The ammonia basal medium of Knight \& Proom (1950) was used to supply ammonia, inorganic salts and trace metals. The medium was supplemented as required with $0.1 \%$ glucose, L-glutamic acid $(1.4 \mathrm{mg} . / \mathrm{ml}$. medium), L-cystine $(0.02 \mathrm{mg} . / \mathrm{ml}$. medium $)$, nicotinic acid $(0.5 \mu \mathrm{g} . / \mathrm{ml}$. medium) and calcium pantothenate $(1 \mu \mathrm{g} . / \mathrm{ml}$. medium $)$. The glucose, amino acids and growth factors were added aseptically at ten times the required concentration after sterilization by Seitz filtration.

\section{RESULTS}

One strain, NCTC 9191 (Ewing, Tanner \& Dennard's (1954) Providence 0 group 10), produced amines, required nicotinic and pantothenic acids as essential metabolites, actively decomposed urea, was indole-positive, vpnegative, MR-weakly positive, $\mathrm{H}_{2} \mathrm{~S}$-positive, did not liquefy gelatin and produced acid with a small bubble of gas from glucose, but did not ferment lactose, maltose, mannitol or sucrose. This strain appeared to be a typical Proteus morganii. The results of the examination of the remaining 55 strains are summarized in Table 1.

For convenience the results are arranged in four groups, but it is not intended to imply that these represent four clearly defined subgroups. Indeed it is clear from Table 1 that there is little correlation between the variable characters. Some of the biochemical reactions, in particular indole production and gas production, were variable. A few strains were plated and individual colonies selected. With some strains both indole-positive and indole-negative variants occurred although repeated tests with the original cultures usually 
gave consistent results. It is possible that with a longer incubation period some strains reported here as indole-negative would have been found to give positive reactions. After plating, some colonies produced anaerogenic cultures and some produced a small bubble of gas with glucose. Repeated tests on the same cultures sometimes gave inconsistent results. Tests for $\mathbf{H}_{2} \mathrm{~S}$ production have recently been reviewed by Clarke (1953) and it seems clear that the conditions of the test can be arranged to produce almost any desired result. The method adopted here has been to test batches of nutrient broth

\section{Table 1. The nutritional and biochemical properties of 55 Providence strains}

Tests

Growth on basal salt medium
supplemented with glucose,
glutamic acid and cystine
Amine production by ninhydrin
test-paper technique
Phenylpyruvic acid reaction
Fermentation reaction with
Glucose
Lactose
Sucrose
Maltose
Mannitol
Indole production
Voges-Proskauer reaction
Methyl red test
Liquefaction of gelatin
Decomposition of urea
Number of strains

\begin{tabular}{cccc}
+ & \multicolumn{2}{c}{ Results } & + \\
+ & + & + & - \\
+ & + & - & + \\
A or ASB & A or ASB & A or ASB & A or ASB \\
- & - & $-(1 . A L)$ & - \\
AL & AL & AL & AL \\
- & $-(1 . A L)$ & $-(2 . A L)$ & - \\
- & - & $-(3 . A L)$ & - \\
+ & - & + & - \\
- & - & - & - \\
$+w$ & $+w$ & $+w$ & $+w$ \\
- & - & - & - \\
- & - & $-(1+)$ & - \\
14 & 8 & 24 & 9
\end{tabular}

$+=$ positive reaction; $-=$ negative reaction; $A$ or $A S B=$ production of acid with or without a small bubble of gas; $\mathrm{L}=$ late; $w=$ weak. The results in brackets show the number of strains giving anomalous reactions.

with selected strains expected to give strongly positive, weakly positive and negative results. Only those batches giving the expected results were used. With this arbitrary method tests for $\mathrm{H}_{2} \mathrm{~S}$ production are often of taxonomic value. Using this technique Proteus species including $P$. morganii give strongly positive tests for $\mathrm{H}_{2} \mathrm{~S}$ within $24 \mathrm{hr}$. but the Providence strains and rettgeri are weakly positive and are usually negative at $24 \mathrm{hr}$., the difference between the two groups being clear-cut.

All strains fermented glucose with or without a small bubble of gas. When gas was formed the amount was similar to that produced by Proteus species. Sucrose was fermented late and, with a few exceptions, lactose, maltose and mannitol were not fermented. All the strains were vP-negative, Mr weakly positive, did not liquefy gelatin and gave a positive phenylpyruvic acid reaction. One strain was urease-positive.

The amine test was positive with 22 of the 55 strains. The results in all cases were clear-cut and reproducible. There was no correlation between amine 
production and the other variable characters within the group. The one urease-positive strain was amine-negative.

Nutritionally the strains formed a homogeneous group. All strains grew in the basal salt medium with glucose added as a possible source of energy and with added amino acids. The amino acid requirements were simple and satisfied by glutamic acid and cystine. Many cultures grew for two or three subcultures in the absence of cystine but required this amino acid for indefinite serial subculture. No vitamins were required as essential metabolites but pantothenate often improved growth and in two instances was nearly essential. The results with these two strains were rather equivocal, and it is probable that they could be maintained in serial subcultures in the absence of pantothenate. Nicotinic acid was not required; indeed, with many strains the addition of nicotinic acid to this simple medium inhibited growth.

\section{DISCUSSION}

Stuart, Wheeler, Rustigian \& Zimmerman (1943), in their studies on the paracolon group of bacteria, described a serologically heterogeneous group of anaerogenic paracolons which they called type 29911. Stuart, Wheeler \& McGann (1946) studied this group in more detail and described six biochemical subgroups which differed slightly in their fermentation reactions with carbohydrates. They included in this group certain other strains which had previously been described in the literature as Shigella wakefield (Berger, 1945) and the Sachs types B 81 and B 105 described as mannitol negative types of Shigella (Sachs, 1943) and it was suggested that type 29911 should be classified as a transitional group without specific or generic status. The resemblance to Proteus was in the fermentation and IMViC reactions, the ability to swarm on $1.0 \%$ agar plates after the strains had been conditioned by many serial subcultures in semi-solid agar, and in the possession of many minor and some major antigens found in the Proteus group. The fact that an occasional strain gave a weak, and an exceptional strain a strong, urease reaction was regarded as additional evidence of the relationship to Proteus.

Kauffmann (1951), in his collected studies on the Enterobacteriaceae, placed type 29911 and related organisms in a new group which he called the Providence group since many of the isolates studied by Stuart et al. (1946) were isolated from the vicinity of Providence (Rhode Island, U.S.A.). Kauffmann (1951) regarded this group as related to Proteus and stated that the only reliable biochemical difference between the Providence and Proteus groups was the failure of the former to decompose urea. Kauffmann (1951) classified rettgeri in the genus Proteus and stated that the only differences between rettgeri and other members of the genus were that rettgeri fermented mannitol and was sodium citrate-positive.

It has been shown by Henricksen \& Closs (1938) and Henricksen (1950) that Proteus species can be differentiated from other enteric organisms by their ability to produce phenylpyruvic acid by oxidative deamination of L-phenylalanine. All Proteus strains were found to give a positive phenylpyruvic acid 
reaction and only an occasional strain of other Enterobacteriaceae gave a weak or exceptionally a strong reaction. Clarke \& Shaw (1954) found that Proteus, rettgeri and Providence cultures gave positive phenylpyruvic acid tests; and only occasional weak reactions were found with other species in the family. A test for the development of alkalinity from leucine was described and found to be specific for Proteus spp. including rettgeri and Providence strains.

Further information is presented here which is of interest in the classification of Proteus and Providence species. Amine production occurred in a substantial minority of the Providence strains studied, whereas previously it had been found only with Proteus species. The spread-over of amine production into the Providence group does not detract from its value as a definitive generic character for the genus Proteus. In a sense it enhances its value since variable amine production occurs only in a group similar to Proteus in other respects.

The Providence group forms a nutritionally homogeneous group requiring for growth only inorganic salts, glutamic acid and cystine. It differs from the Proteus species in that it does not require nicotinic acid and from rettgeri in that this species will grow on inorganic salts and glucose (Proom \& Woiwod, 1951). Proteus, Providence and rettgeri have three distinct basal nutritional patterns, and these patterns should be considered in relationship to their classification. The emphasis to be placed on nutrition in classification is partly a matter of opinion but more properly depends on the extent to which the patterns are characteristic and constant within the group studied.

The genus Proteus can be defined on the basis of a large number of characters. The characters differentiating this group from other Enterobacteriaceae were given by Proom \& Woiwod (1951) as the ability to swarm on nutrient agar, the fermentation of glucose, but not lactose or mannitol, with production of a small volume of gas, decomposition of urea, marked $\mathrm{H}_{2} \mathrm{~S}$ production, amine production and a nutritional requirement for nicotinic acid. To these may be added a positive phenylpyruvic acid reaction. The loss of one or more of these characters would not necessarily exclude an organism from the genus and the possession of one or more of these characters would not justify its inclusion. The genus is defined on the pattern basis, that is on the assessment of as large a number of characters as possible, and then it represents a clearly defined group.

As soon as the generic definition is widened difficulties occur. The inclusion of rettgeri in the genus invalidates five of these characters, i.e. the production of small volumes of gas with glucose, failure to ferment mannitol, marked $\mathrm{H}_{2} \mathrm{~S}$ production, amine production and a nutritional requirement for nicotinic acid. The generic definition then rests on a somewhat ill-defined biochemical pattern, marked urease reaction, positive phenylpyruvic acid reaction and the development of an alkaline reaction with leucine. The last two tests were not considered when rettgeri was placed in the genus Proteus by Rustigian \& Stuart (1943). If the Providence group is included then the generic definition becomes even more difficult. The biochemical pattern becomes more obscure; urease production is no longer an essential character and only a positive phenylpyruvic acid reaction and the development of an alkaline reaction with 
leucine remain. Positive phenylpyruvic acid reactions are known to occur in a minority of other strains in the family.

Because of a commendable reluctance to establish new genera, attempts are often made to 'press-gang' newly described species into existing genera and widen the generic definition. It is argued here that if the generic definition of Proteus is widened to include the Providence group and rettgeri then the definition becomes so vague that it is almost meaningless. It seems reasonably clear that the only alternative is to establish a new genus to include Providence and rettgeri.

My thanks are due to Mr E. Harris and Miss G. M. Trim for technical assistance.

\section{REFERENCES}

Berger, F. M. (1945). A new non-mannitol-fermenting dysentery organism of the Flexner group. J. Hyg., Camb. 44, 116.

Christensen, W. B. (1946). Urea decomposition as a means of differentiating Proteus and paracolon organisms from each other and from Salmonella and Shigella types. J. Bact. 52, 461.

Clarke, P. H. (1953). Hydrogen sulphide production by bacteria. J. gen. Microbiol. 8, 397.

Clarke, P. H. \& Shaw, C. (1954). Biochemical tests for Proteus and Providence cultures. J. gen. Microbiol. 10, i.

Ewing, W. H., Tanner, K. E. \& Dennard, D. A. (1954). The Providence group: An intermediate group of enteric bacteria. J. infect. Dis. 94, 134.

HenRICKSEN, S. D. (1950). A comparison of the phenylpyruvic acid reaction and the urease test in the differentiation of Proteus from other enteric organisms. J. Bact. 60, 225.

Henricksen, S. D. \& Closs, K. (1938). The production of phenylpyruvic acid by bacteria. Acta path. microbiol. scand. 15, 101.

Kauffmann, F. (1951). Enterobacteriaceae. Copenhagen: Ejnar Munksgaard.

Knight, B. C. J. G. \& Proom, H. (1950). A comparative survey of the nutrition and physiology of mesophilic species in the genus Bacillus. J.gen. Microbiol. 4, 508 .

Kovács, N. (1928). Eine vereinfachte Methode zum Nachweis der Indolbildung durch Bakterien. Z. ImmunForsch. 55, 34.

O'Meara, R. A. Q. (1931). A simple, delicate and rapid method of detecting the formation of acetyl methylcarbinol by bacteria fermenting carbohydrate. J. Path. Bact. 34, 401.

Proom, H. \& Worwod, A. J. (1951). Amine production in the genus Proteus. J. gen. Microbiol. 5, 930.

Rustigian, R. \& Stuart, C. A. (1943). Taxonomic relationships in the genus Proteus. Proc. Soc. exp. Biol., N.Y. 53, 241.

SACHS, A. (1943). A report of an investigation into the characteristics of new types of non-mannitol-fermenting bacilli isolated from cases of bacillary dysentery in India and Egypt. J. R. Army med. Cps, 80, 92.

STAMP, Lord \& STONE, D. M. (1944). An agglutinogen common to certain strains of lactose- and non-lactose-fermenting coliform bacilli. J. Hyg., Camb. 43, 266.

Stuart, C. A., Wheeler, K. M. \& McGanN, V. (1946). Further studies on one anaerogenic paracolon organism type 29911. J. Bact. 52, 431.

Stuart, C. A., Wheeler, K. M., Rustigian, R. \& Zimmerman, A. (1943). Biochemical and antigenic relationships of the paracolon bacteria. J. Bact. 45, 101. 